Biogerontology, volume 23, issue 1: https://doi.org/10.1007/s10522-022-09951-4

\title{
Geroscience: the need to address some issues
}

\author{
Eric Le Bourg \\ Eric Le Bourg, Centre de Recherches sur la Cognition Animale (CRCA), Centre de Biologie \\ Intégrative (CBI Toulouse), Université de Toulouse, CNRS, UPS, Toulouse, France \\ e-mail address: eric.le-bourg@univ-tlse3.fr \\ Orcid iD: https://orcid.org/0000-0001-5578-025X
}

\begin{abstract}
The geroscience field hypothesises that it could be possible to delay all age-related diseases by delaying the ageing process itself, and not by curing each age-related disease individually. While this perspective is attracting, some issues provisionally prohibit to fully adhere to it, such as the risk for some over-enthusiasm or scientism and the tendency to accept that results observed in animal models can be easily translated in human beings. Particularly, it is not clear whether geroscience plans to delay or suppress ageing, or if healthspan could become very close to lifespan. This article lists some of these issues, in the hope that supporters of geroscience will grasp them to allow a full development of the field, and argues that geroscience should avoid reductionist approaches not taking into account social and behavioural complex aspects of human ageing.
\end{abstract}

Key-words: geroscience — healthspan — lifespan 


\section{Introduction}

The word geroscience is now widely used by many authors to best describe the theoretical ground of their research on ageing. This concept has been summarised by Sierra (2016) as follows: 1- "the ultimate goal of biomedical research is to improve the quality of life in humans", 2- "chronic diseases and conditions of the elderly represent the main hurdle toward reaching that goal", 3"because aging is malleable (at least in many animal models) and aging is also the main risk factor for those diseases and conditions, then addressing the basic biology of aging is likely to provide a better payoff than addressing diseases one at a time".

In other words, rather than treating each age-related disease separately, such as cancer (Farelly, 2021), it would be better to delay the whole ageing process, which would increase healthspan, i.e., the period of life without major diseases and disability. This idea seems sound and was considered as giving rise to the "new field of geroscience" (Burch et al., 2014). However, this geroscience concept is plagued with some issues maybe prohibiting full benefits of its use. The purpose of this article is to address them in the hope to favour a full growing of the field.

\section{Delaying ageing to increase healthspan: but what about lifespan?}

Sierra (2016) wrote that even if "recent advances have made the idea of addressing the role of aging as the major risk factor for most chronic diseases closer to being feasible, the idea itself is not new". Indeed, Sierra (2016) cited a report of the National Science Foundation (Neugarten and Havighurst, 1977), where Havighurst and Sacher (1977) wrote that "if this aging process could be understood, it might be treated so as to delay the process, and therefore give people more years of health and vigor before they become vulnerable to the common diseases of old age". This echoes Hayflick's (2021) lamentations that, for decades, "the greatest risk factor for age associated diseases is neglected in favor of research on the diseases themselves". However, this idea was even stated more than 120 years ago as shown by the book of the French author Jean Finot, where he wrote that "modern biology has conceived the heroic project of directly attacking old age itself" and that "these remedies once known we shall by those means alone defer the approach of senility" (Finot, 1900: pages 109 and 113 in the English book). Therefore, it is not sure that "there has been a recent paradigm shift away from attempting to cure specific diseases, the 'whack-a-mole' approach, to that of understanding and slowing biological aging - the underlying cause of diseases of aging - as embraced by the emerging interdisciplinary field of Geroscience" (Epel, 2020). It is not risky to say that every biogerontologist is convinced that, if it were possible to delay the ageing process, it could also be possible to delay age-related diseases, but past gerontologists probably shared the same opinion. 
What appears to be new, however, is telling that geroscience bears the hope that "addressing aging (as opposed to addressing one disease at a time) leads to robust elderly individuals, not sick ones... In that scenario, the 'new elderly' will not produce an undue burden on the health systems" (Sierra, 2016). One thus understands that people will live longer, with healthspan close to lifespan: people enjoy a good health for life and, one day, they die, like in the Aldous Huxley's (1932) Brave new world novel, but not when reaching 60 years of age like in the novel. This idea of dying at old age without being subjected to the ageing process is highly speculative, because it would mean that people die mainly from accidents or diseases not linked to age and thus, by chance, could reach very old ages, possibly beyond the current maximal lifespan of 122 years. A less extreme view was given by Sierra et al. (2021) who stated that "resulting infirmities associated with old age will be compressed into a shorter time frame" but the same authors were more extreme when they added that "by implementing anti-aging interventions throughout life, perhaps we can anticipate a delay or suppression of the development of disease in older adults". One thus understands that if the development of diseases is suppressed at old age, ageing is suppressed, too, and people die from accidents or diseases not linked to age or maybe for unknown reasons. Blagosklonny (2021) has criticised the idea of increasing healthspan without increased lifespan, arguing that "in this scenario, cancer kills an organism in a matter of minutes, instead of months. This is impossible". As this author entitled his article "The goal of geroscience is life extension" his view is that, thanks to geroscience, people could die later with a longer healthspan, healthspan being shorter than lifespan as today: the ageing process is delayed, not suppressed.

Therefore, there is a hard debate among geroscientists: do they conclude that healthspan increase will make lifespan to increase, or that healthspan will be close to lifespan? Under the first hypothesis, ageing is delayed and people live longer, but the last part of the life is still loaded with age-related diseases: ageing is not suppressed. Under the second one, people die suddenly without being confronted to age-related diseases: ageing is actually suppressed, as observed in Pacific salmons, science-fiction movies and novels.

Authors supporting the geroscience concept should describe more extensively the transition between healthspan and death if geroscience would be able to delay or suppress ageing.

\section{Over-enthusiasm for geroscience is risky for geroscience itself}

The geroscience concept has given rise to some writings that give the feeling that we are near discovering the means to delay, and even reverse, the ageing process by relying on biology. For instance Mahmoudi et al. (2019) wrote that "a key question is whether ageing of cells, tissues and organisms can be reversed or 'rejuvenated' rather than simply delayed". In France, a new institute 
named "Restore: a geroscience and rejuvenation research center" (https://restore-lab.fr) makes use of the word "réjuvénation" in French, but this word does not exist in this language and has thus no meaning. Using the word rejuvenation makes that the lay public and other scientists may conclude that serious authors propose to rejuvenate organisms and people, which is not so far from the speech of charlatans claiming they can make you live for many extra-years, if not reverse the ageing process, provided you pay for their miraculous recipes. The history of gerontology is replete with such claims in past decades, and even centuries (Stambler, 2020): today, transhumanists (Kurzweil and Grossman, 2010) and biotechnological companies (Brooker, 2015) trying to attract investors rely on the same hopes. Lee and Kaeberlein (2018) published survival curves of Black, Hispanic, and White US people, together with an "idealized human survival by assuming that the maximum human lifespan is close to what we've already witnessed". The authors discuss that "to achieve this ideal, an intervention (or combinations of interventions) would need to extend median lifespan by $\sim 30 \%$ in the longest-lived populations and by closer to $40 \%$ in the shortest-lived populations (black males and females)", median lifespan reaching 110 and 115 years in men and women (Fig. 3 in Lee and Kaeberlein, 2018). Indeed, one may wonder whether it is sound to hypothesise that one half of US people could live beyond 110 or 115 years of age, thanks to geroscience (Le Bourg, 2012).

Therefore, some colleagues make use of a concept, rejuvenation, that is highly open to criticism when used at the organismal level because, today, there is not a single means to rejuvenate an animal or a human: The curious case of Benjamin Button does not exist in real life. This way, they give the false hope that the end of ageing is coming soon, as some scientists in past decades did (Stambler, 2020), which could make other scientists reluctant when reading geroscience papers. In addition, they propose that the lifespan of most people could approach that of Jeanne Calment, the 122-years record-woman. It is not sure that over-enthusiasm is of help to promote the geroscience concept.

\section{Geroscience: at risk of scientism?}

Geroscience brings the hope that, relying on biology, it could be possible to delay age-related diseases by delaying the ageing process, to the point that some articles consider that the lifespan differences among people are mainly linked to diseases and not to social differences. For instance, Lee and Kaeberlein (2018) wrote that "comparing black and white populations..., the biggest difference in causes of death between these groups is due to diabetes" and that "if healthspan interventions are similarly equitable in human populations as they are in model systems, we may see the largest benefits in health among those with the shortest lifespans, particularly black populations. Decreased health disparities that result from healthspan intervention can be thought of 
as another important longevity dividend". However, considering that, because diabetes mainly explains the lifespan difference between Black and White US people, removing diabetes would strongly erase lifespan differences between them is accepting that diabetes prevalence is not linked to social differences. There are many social reasons explaining why Black US people have a higher prevalence of diabetes, a higher infant mortality, and live shorter than White US people, but geroscience is unable to suppress social and racial inequalities even if the magic pill removing diabetes were to be discovered. This point has been emphasised by Epel (2020) stating that "poor behaviors can override effects of protective pharmaceuticals. A common example of this is that people still develop diabetes while taking metformin due to overeating a western junk food diet. Improving health behaviors can best be prioritized and implemented in the context where basic social needs are met."

In the past decades, the human ageing process has been delayed thanks to sanitation, medical and social progress, with the result that 60 -year-old people in wealthy countries are highly different from people of the same age who lived one century ago. Clearly enough, medicine was of help, but was not the main explaining factor and, today, ageing differences among people could not be erased by relying mainly on biology. The issue of social determinants of human ageing has been addressed by Crimmins (2020), who emphasised that "social factors are strongly related to physical and cognitive functioning and multimorbidity" and that "scientific advances will be made when biological approaches incorporate some of the concepts in the social hallmarks of aging in experimental design to test pathways based on what has been shown to promote earlier aging in humans". In other words, it would be risky for the geroscience initiative to consider that biological remedies could erase ageing differences linked to social factors. This would be a kind of scientist illusion, but science, medicine, and technology are not able to resolve all human issues.

The good news is that the article of Sierra et al. (2021) gathers social and behavioural scientists, as well as demographers and biologists, in an attempt to take into account the interconnection of biological, behavioural, psychological, and social factors shaping the ageing fate of the very people. These authors rightly state that translation of results observed in animal models in humans will be a hard challenge, by contrast with other scientists who seemingly think that this is an easy process (see the next paragraph).

\section{Geroscience should not forget evolutionary biology}

A risk for the credibility of the geroscience field would be to consider that results gathered on animal models can be "translated", more or less easily, in humans. Beyond social and other factors discussed above, this reasoning ignores that life-histories differ among species (see Le Bourg, 2016) 
and that mice are not small humans (Demetrius, 2005). This can make that authors cite studies to support the idea that results on animal models can be translated to humans, while such conclusions are not reached when reading these very studies.

For instance, Lee and Kaeberlein (2018) report that hypoxia increases lifespan in the worm Caenorhabditis elegans and "may explain longevity among people living at high altitude". Indeed, if hypoxia would increase lifespan in both worms and humans, it would be an outstanding result to the very least. Yet, the cited study ( $\mathrm{Li}$ et al., 2017) cannot be used to support any hypothesis. The authors, using the 2010 Chinese census, observed that the proportion of centenarians or of $>91$ yearold people was higher in the Tibetan than in the Han populations. However, the age structure of a population cannot be used to conclude about lifespan because this structure is the result of past events: births, migrations, deaths (discussion in Scheidel, 2001). To give an example, comparing the age structure of people living in a retirement home (no births, in-migration but no out-migration, many deaths) and in a university campus (births, in- and out-migrations, few deaths) cannot allow to conclude that lifespan is higher in a retirement home, even if ages at death are older in the retirement home. Thus, the Li et al.'s study (2017) cannot be used to claim that Tibetans live longer than Hans. In addition, even if Tibetans would live longer, it could obviously not be concluded that, because of the high altitude in Tibet, hypoxia increases human longevity. While it can be understood that Lee and Kaeberlein (2018) had no background in demography, and thus cited this flawed study, it remains that they accepted the idea that homeothermic humans and poikilothermic worms share the same metabolism and thus that results observed in worms will also be observed in humans.

Similarly, Mahmoudi et al. (2019), elaborating on the lifespan increases linked to dietary restriction in mice, wrote that "fasting-mimicking diet improves body weight, blood pressure, cholesterol and IGF1 levels and other physiological readouts when applied in humans" and cited a paper reporting such results. However, this study (Wei et al., 2017) stratified subjects by risk-factor levels and showed that the fasting-mimicking diet had positive effects mainly in people with risk factors. Obese people, for instance, benefited from restricting their diet, which is not surprising but also shows that diet restriction had no clear effect in normal-weight people. In such conditions, it is going too far when concluding that diet restriction has positive effects in humans, without making any distinction between normal-weight and obese people: showing that diet restriction could help to treat pathologies linked to obesity is not the same as showing that it could delay the normal ageing process.

Thus, diet restriction can increase lifespan in some species (Le Bourg, 2010, 2018), or some genotypes in mice (Liao et al., 2010, but see Unnikrishnan et al., 2021), but it is not a cue to 
conclude that diet restriction could have similar effects in humans or decrease risk factors for agerelated diseases, because mice and humans do not face food shortage by relying on the same strategy (Demetrius, 2005; Le Bourg, 2016).

\section{Conclusions}

Geroscience is an ambitious initiative planning to translate in humans positive effects of various treatments in animal models, in the hope to increase healthspan and delay age-related diseases, this without the need to cure each disease. Under this approach, physicians and scientists are proposed to take into account the whole ageing process of people when trying to fight an agerelated disease, and not only this very disease. However, the irony is that some authors made the point that "geroscience has not heretofore incorporated behavioral or social-science methods or findings into its agenda" (Moffitt, 2020). In other words, some authors think that the holistic approach of geroscience still fails to be really holistic. The most extreme reductionist view is maybe that of Hayflick (2021), advocating for basic research on ageing, who stated that "the greatest risk factor for the leading cause of death is a problem in physics, not biology" and that "the net loss of functional molecules and/or the accumulation of unrepaired dysfunctional molecules are necessary and sufficient stochastic events to cause aging processes in both living forms and in inanimate objects. The cause is the 2nd Law of Thermodynamics and increasing entropy". One thus understands that biology does not really exist and, because ageing is explained by physics, inanimate objects and living beings are, finally, very similar.

We may feel that the geroscience initiative is still in its infancy and that its concepts will still evolve. In this way, it would be needed to discard some issues that could plague the development of geroscience and this paper is an attempt to list some. Not doing so could have the consequence that other biogerontologists, physicians, and colleagues concerned with the ageing process could conclude that geroscience is maybe only a new way for some authors to claim that some remedies can suppress ageing, particularly those they are studying, and to attract funding. It is too early to state whether the geroscience initiative will success in delaying (or suppressing?) age-related diseases, and thus to delay (or suppress?) ageing, by translating in humans results observed in animal models. However, it is maybe useful to recall that, in the past, some authors claimed to have discovered the cause of ageing, and thus probably the remedy. For instance, Harman (1998) wrote that there is a "growing consensus that free radical reactions are a major cause of ageing, possibly the only one". If geroscience is only replacing the claim that antioxidants can delay ageing by the claim that metformin, rapamycin, or any other molecule, can do the same, in accordance with the “exciting promise that a single 'geroprotective' therapy that slows aging could delay multiple age- 
related diseases" (Sierra et al., 2021), geroscience would be not more than the modern refreshing of an old idea, that of the long-life elixir.

Geroscience has been promoted by "hard" biologists that are often not comfortable with other domains, such as demography, social sciences, or evolutionary biology. This may explain some issues listed above but the good news is that they can be easily overcome, because "suggestions for geroscience research" (Crimmins, 2020) have already been stated. Particularly, Moffitt (2020) put a premium on collaborations with other scientists.

Therefore, this article does not argue that new breakthroughs in biology, medicine, and technology will be unable to delay ageing, or, on the contrary, that these breakthroughs will make healthspan becoming very close to lifespan, which could also increase up to the current maximal lifespan, or even higher: time will tell. However, it is argued that colleagues supporting the "optimistic side", the geroscientists, should take care to clarify some issues of the geroscience field, and this article has listed some.

Even if some colleagues are dubious about the eventual success of the geroscience initiative, the best move is maybe to say "let a hundred flowers bloom" until geroscientists bring to the fore positive results. If they fail, it will be one of the innumerable failures of science, if they success, even partly, it will be a major result towards a better knowledge of the ageing process.

\section{References}

Blagosklonny MV (2021) The goal of geroscience is life extension. Oncotarget 12:131-144

Brooker K (2015) Google ventures and the search for immortality (March 9 $9^{\text {th }}, 2015$ ) https://www.bloomberg.com/news/articles/2015-03-09/google-ventures-bill-maris- investingin- idea-of-living-to-500

Burch JB, Augustine AD, Frieden LA, Hadley E, Howcroft TK, Johnson R, Khalsa PS, Kohanski RA, Li XL, Macchiarini F, Niederehe G, Oh YS, Pawlyk AC, Rodriguez H, Rowland JH, Shen GL, Sierra F, Wise BC (2014) Advances in geroscience: impact on healthspan and chronic disease. J Gerontol A Biol Sci Med Sci 69: Suppl1:S1-S3

Crimmins EM (2020) Social hallmarks of aging: suggestions for geroscience research. Ageing Res Rev 63:101136

Demetrius L (2005) Of mice and men. When it comes to studying ageing and the means to slow it down, mice are not just small humans. EMBO Rep 6:S39-S44

Epel ES (2020) The geroscience agenda: toxic stress, hormetic stress, and the rate of aging. Ageing Res Rev 63:101167

Farrelly C (2021) Responsible biology, aging populations and the 50 $0^{\text {th }}$ anniversary of the "War on 
Cancer”. Biogerontology 22:424-440

Finot J (1900) La philosophie de la longévité. Paris: Librairie C. Reinwald. (in English: The philosophy of long life: London: Richard Clay \& Sons, 1909:

https://archive.org/details/philosophyoflong00finouoft/mode/2up)

Harman D (1998) Extending functional life span. Exp Geront 33:95-112

Havighurst RJ, Sacher GA (1977) Prospects of lengthening life and vigor. In: Neugarten BL, Havighurst RJ, eds., Extending the human life span: social policy and social ethics. National Science Foundation, Arlington, VA, pp. 13-18

Hayflick L (2021) The greatest risk factor for the leading cause of death is ignored. Biogerontology 22:133-141

Huxley AL (1932) Brave new world. London: Chatto \& Wendus

Kurzweil R, Grossman T (2010) Bridges to life. In: Fahy GM, West MD, Coles LS, Harris SB, eds.

The future of aging. Pathways to human life extension. Berlin: Springer, 2010:3-22

Le Bourg E (2010) Predicting whether dietary restriction would increase longevity in species not tested so far. Ageing Res Rev 9:289-297

Le Bourg E (2012) Forecasting continuously increasing life expectancy: what implications? Ageing Res Rev 11:325-328

Le Bourg E (2016) The somatotropic axis may not modulate ageing and longevity in humans. Biogerontology 14:421-429

Le Bourg E (2018) Does calorie restriction in primates increase lifespan? Revisiting studies on macaques (Macaca mulatta) and mouse lemurs (Microcebus murinus)? BioEssays 40:1800111

Lee MB, Kaeberlein M (2018) Translational geroscience: from invertebrate models to companion animal and human interventions. Transl Med Aging 2:15-29

Liao CY, Rikke BA, Johnson TE, Diaz,V, Nelson JF (2010) Genetic variation in the murine lifespan response to dietary restric- tion: from life extension to life shortening. Aging Cell 9: $92-95$

Li Y, Wang MS, Otecko NO, Wang W, Shi P, Wu DD, Zhang YP (2017) Hypoxia potentially promotes Tibetan longevity. Cell Res 27:302-305

Mahmoudi S, Xu L, Brunet A (2019) Turning back time with emerging rejuvenation strategies. Nat Cell Biol 21:32-43

Moffitt TE (2020) Behavioral and social research to accelerate the geroscience translation agenda. Ageing Res Rev 63:101146

Neugarten BL, Havighurst RJ (eds.) (1977) Extending the human life span: social policy and social 
ethics. National Science Foundation, Arlington, VA

Scheidel W (2001) Roman age structure: evidence and models. J Roman Stud 91:1-26

Sierra F (2016) The emergence of geroscience as an interdisciplinary approach to the enhancement of health span and life span. Cold Spring Harb. Perspect Med 6:a025163

Sierra F, Caspi A, Fortinsky RH, Haynes L, Lithgow GJ, Moffitt TE, Olshansky SJ, Perry D, Verdin E, Kuchel GA (2021) Moving geroscience from the bench to clinical care and health policy. J Am Geriatr Soc 69:2455-2463

Stambler I (2020) History of life-extensionism. In: Rattan SIS, Barbagallo M, Le Bourg E, eds. Encyclopedia of biomedical gerontology, Vol. 2. London: Academic Press, 2020:228-237

Unnikrishnan A, Matyi S, Garrett K, Ranjo-Bishop M, Allison DB, Ejima K, Chen X, Dickinson S, Richardson A (2021) Reevaluation of the effect of dietary restriction on different recombinant inbred lines of male and female mice. Aging Cell 20:e13500

Wei M, Brandhorst S, Shelehchi M, Mirzaei H, Cheng CW, Budniak J, Groshen S, Mack WJ, Guen E, Di Biase S, Cohen P, Morgan TE, Dorff T, Hong K, Michalsen A, Laviano A, Longo VD (2017) Fasting-mimicking diet and markers/risk factors for aging, diabetes, cancer, and cardiovascular disease. Sci Transl Med 15:eaai8700 